\title{
Groundstate $\mathrm{OH}$ masers associated with Herbig-Haro objects
}

\author{
A. de Witt and D. P. Smits \\ Department of Mathematical Sciences, University of South Africa, Pretoria, 0003, South Africa \\ email: aletdewitt@webmail.co.za,dps@astro.unisa.ac.za
}

\begin{abstract}
Jets from newly-formed stars plow into the surrounding ISM, creating nebulous regions known as Herbig-Haro $(\mathrm{HH})$ objects. Signatures of C-type shocks have been found in many HH objects. Models of shock-excited 1720-MHz OH masers associated with SNRs suggest that the masers could be signposts of C-type shocks. We have conducted a $1720 \mathrm{MHz}$ survey towards a number of $\mathrm{HH}$ objects to see if any shock-excited masers occur in association with these objects. Some results of our search are reported. When present, the $1720-\mathrm{MHz}$ OH line can occur as emission, absorption or as $\mathrm{P}$ Cygni profiles. $\mathrm{OH}$ mainline emission is found in many of our sources. We have identified several potential 1720-, 1665- and 1667-MHz OH masers.
\end{abstract}

Keywords. ISM: Herbig-Haro objects, ISM: jets and outflows, masers, line: profiles

\section{Introduction}

Herbig-Haro $(\mathrm{HH})$ objects are shock-excited nebulae identified from characteristic line ratios in their optical spectra. They are associated with outflows, usually in the form of jets, often (but not exclusively) from protostars with $M_{*} \leqslant 4 M_{\odot}$. When these jets impact the interstellar medium (ISM), bow-shaped structures are formed which show signatures of J-type shocks at the apex and C-type shocks on the wings. For example, modelling of HH 7 by Smith, Khanzadyan \& Davis (2003) successfully explained their near infrared observations of $2.12 \mu \mathrm{m} \mathrm{H}_{2}$ line emission in terms of a C-type shock on the wings of the bow structure.

About 10\% of known Galactic supernova remnants (SNRs) have been found to support 1720-MHz OH masers at their shock interface. Lockett, Gauthier \& Elitzur (1999) modelled these regions and showed that under a restrictive set of physical conditions, $1720-\mathrm{MHz} \mathrm{OH}$ masers can be collisionally excited in a C-type shock formed when a SNR interacts with a dense molecular cloud within the ISM. Observationally the masers have been found to occur along a ridge where the $2.12 \mu \mathrm{m} \mathrm{H}_{2}$ line emission peaks. Based on the tight constraints under which the masers form, Lockett et al. (1999) suggested that $1720 \mathrm{MHz} \mathrm{OH}$ masers could be an important signpost of C-type shocks. This report presents some results of a survey carried out on the $100 \mathrm{~m}$ Effelsberg and Greenbank telescopes to search for $1720-\mathrm{MHz} \mathrm{OH}$ masers towards $\mathrm{HH}$ objects.

\section{Observations}

\subsection{Source selection}

Based on SNR statistics, only $10 \%$ of SNRs searched have $1720-\mathrm{MHz} \mathrm{OH}$ masers. If the same statistics apply to $\mathrm{HH}$ objects, a large number of sources need to be surveyed to find at least a few maser detections. Furthermore, shock-excited masers are not as intense as those in H II regions and therefore a survey with high sensitivity is required. The most 
efficient way to search a large number of sources for masers is to use single-dish telescopes with large collecting surface areas. Sources with positive detections can be re-observed with interferometers to confirm the presence of masers.

A sample of HH objects were selected from Reipurth's catalogue (Reipurth, 1999) to search for $1720-\mathrm{MHz} \mathrm{OH}$ emission. The nearest $\mathrm{HH}$ objects were our primary targets, as they should have the strongest intensity. Sources for which a case has been made in the literature that C-type shocks are present were also included in our list. A total of $231 \mathrm{HH}$ objects have been observed with either the Greenbank or Effelsberg telescopes. Sometimes more than one object fell within the $7^{\prime}$ beam of the telescope so that the number of objects observed exceeds the number of pointings.

\subsection{Greenbank $100 m$ Telescope}

Observations using the Greenbank 100m telescope were performed on 04 and 07 February 2005. We simultaneously observed the $1720-\mathrm{MHz} \mathrm{OH}$ satellite line and the $\mathrm{OH}$ groundstate mainlines at 1665 and $1667 \mathrm{MHz}$ in both LCP and RCP towards 45 positions. With a bandwidth of $12.5 \mathrm{MHz}$ this gave a velocity coverage of $\pm 1090 \mathrm{~km} \mathrm{~s}^{-1}$ at a resolution of $\pm 0.13 \mathrm{~km} \mathrm{~s}^{-1}$. The total integration time per source was 15 mins per pointing, giving a typical noise level of $\sim 30 \mathrm{mJy}$.

\subsection{Effelsberg 100m Telescope}

Observations using the Effelsberg $100 \mathrm{~m}$ telescope were performed on 28 February, 02 March, 05 May and 20 May 2005. Only the $1720-\mathrm{MHz} \mathrm{OH}$ line was observed in both LCP and RCP towards 74 positions. With a bandwidth of $1.56 \mathrm{MHz}$, the 1024 channel correlator gave a velocity coverage of $\pm 84 \mathrm{~km} \mathrm{~s}^{-1}$ at a resolution of $\pm 0.53 \mathrm{~km} \mathrm{~s}^{-1}$ after folding the spectra. Integration times varied between 10 and 15 mins per pointing, giving a typical noise level of $\sim 40 \mathrm{mJy}$.

\section{Results}

For the Greenbank data at $1720 \mathrm{MHz}$ there were 18 non-detections, 16 pointings show lines in emission, 4 have absorption and 7 have $\mathrm{P}$ Cyg-type profiles. Two of the $\mathrm{P}$ Cyg profiles have blue-shifted absorption and red-shifted emission indicating infall rather than outflow. Line widths of $\Delta v \sim 0.5-3.5 \mathrm{~km} \mathrm{~s}^{-1}$ are consistent with those of the $1720 \mathrm{-MHz}$ $\mathrm{OH}$ masers in SNRs. These are broader than would be expected for a single maser spot but could be multiple lines unresolved in velocity.

A surprising result is that the Greenbank data show 1665- and $1667-\mathrm{MHz} \mathrm{OH}$ emission lines at all but 4 of the pointings, 14 of which have no corresponding $1720 \mathrm{MHz}$ lines. None of the sources show unambiguous $1720-\mathrm{MHz} \mathrm{OH}$ maser emission, i.e. $1720 \mathrm{MHz}$ in emission and $1667 \mathrm{MHz}$ in absorption.

For the $\mathrm{OH}$ mainlines, optically thin thermal emission has a flux density ratio $F(1665)$ : $F(1667)=5: 9$, while for optically thick conditions the ratio is $1: 1$. If observed flux densities have a ratio $F(1667)$ : $F(1665)$ outside this range, this is suggestive of nonthermal emission such as a maser. A number of our sources have non-thermal line ratios for the mainlines. All of the sources with P Cyg profiles at $1720 \mathrm{MHz}$ have non-thermal mainline ratios less than 1 , indicating that $F(1665)>F(1667)$.

In Fig. 1 some examples of the Greenbank data are presented. HH33 is an example of a back-to-front P Cyg profile. The $1720 \mathrm{MHz}$ absorption peak corresponds in velocity to that of one peak in the 1665 and $1667 \mathrm{MHz}$ spectra, while the $1720 \mathrm{MHz}$ emission peak is offset from that of the mainlines by $\sim 1 \mathrm{~km} \mathrm{~s}^{-1}$. HH 167 and $\mathrm{HH} 279$ have no detectable features at $1720 \mathrm{MHz}$, but have $F(1667): F(1665)$ of 2.40 and 2.80 respectively. HH 200 

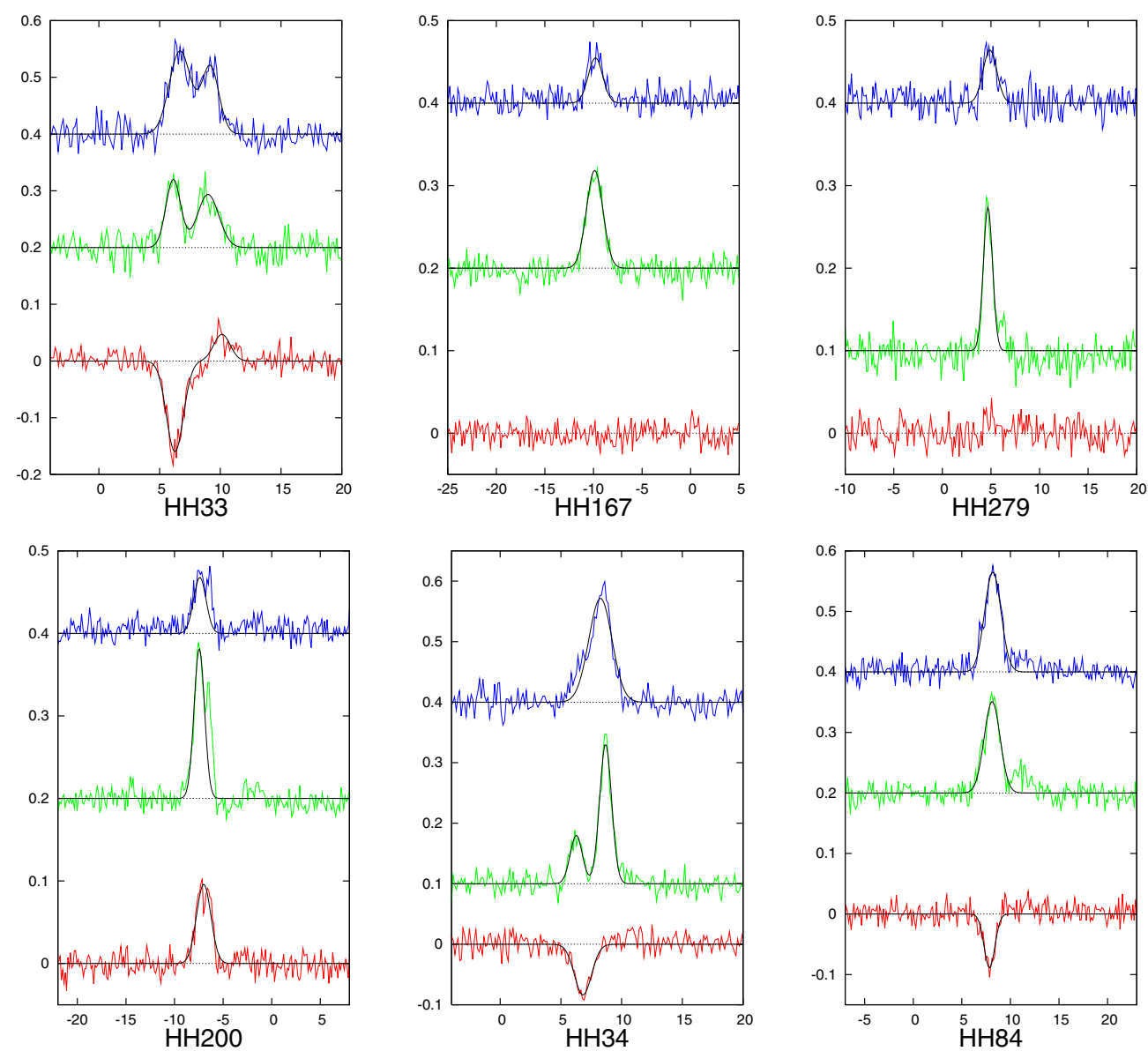

Figure 1. Some example spectra from Greenbank, plotting flux density in Jy against velocity in $\mathrm{km} \mathrm{s}^{-1}$. In each panel from top bottom the 1665-, $1667-$ and $1720-\mathrm{MHz}$ data are plotted together with a fitted Gaussian.

appears to have two peaks, both of which are narrow, but they cannot be resolved when fitting Gaussians. In this object, $1720 \mathrm{MHz}$ appears in emission and the mainlines have a flux ratio $F(1667): F(1665)=2.60$. HH 34 and $\mathrm{HH} 84$ are examples in which the $1720 \mathrm{MHz}$ is in absorption while the mainlines are in emission. In $\mathrm{HH} 34$ there could be structure in the blue wing of the $1665 \mathrm{MHz}$ line which we cannot resolve; the fitted Gaussians for the $1665 \mathrm{MHz}$ line and the red peak in the $1667 \mathrm{MHz}$ spectrum have a thermal line ratio. The velocity of the $1720 \mathrm{MHz}$ absorption dip is offset from the blue peak in $1667 \mathrm{MHz}$ by $v \sim 0.6 \mathrm{~km} \mathrm{~s}^{-1}$. HH 84 has mainlines with $F(1667): F(1665)=0.88$. The fitted Gaussians all peak at slightly different velocities.

The $1720 \mathrm{MHz}$ data from Effelsberg have 48 non-detections, 10 sources have emission, 11 have absorption and 5 have $\mathrm{P}$ Cyg-type profiles. 1 out of 5 sources with $\mathrm{P}$ Cyg profiles indicate infall rather than outflow. Gaussian fits to the line profiles show similar properties to those from the Greenbank data. 


\section{Discussion}

With flux densities between 0.03 and $0.4 \mathrm{Jy}$, all the lines are weak, particularly compared to the $\mathrm{OH}$ maser intensities found in star-forming regions. Weak $\mathrm{OH}$ emission is present throughout much of the Galaxy, but in most cases it is from extended sources with the 1665- and 1667-MHz lines having fluxes indicative of thermal emission. Many of the sources we have found have mainline flux ratios indicative of non-thermal emission.

Using the Effelsberg telescope in January 1986, Wouterloot, Brand \& Fiegel (1993) did not report any 1665- or $1667-\mathrm{MHz} \mathrm{OH}$ emission to a limit of $<50 \mathrm{mJy}$ from $\mathrm{HH} 288$, whereas we found a $60 \mathrm{mJy}$ line at $1665-\mathrm{MHz}$ with $\mathrm{F}(1665)>\mathrm{F}(1667)$. According to the Lockett et al. (1999) theory, shock-excited $1720-\mathrm{MHz}$ OH masers are always weak and hence never saturate. Variability on time scales of weeks to months is quite possible when dealing with unsaturated masers.

Argon, Reid \& Menten (2003) reported weak OH masers in the W3 OH region that appear to form at the edges of the outflow of a protostellar source, the Turner-Welch object, which also drives $\mathrm{H}_{2} \mathrm{O}$ masers. They suggest that protostellar outflows may be an important mechanism for the production of weak $\mathrm{OH}$ masers when no ionizing stars are present. They point out that it is possible, due to bandwidth and sensitivity limitations, that most surveys could have missed weak maser features. Some of the sources mapped by Forster \& Caswell (2000) containing both $\mathrm{OH}$ and $\mathrm{H}_{2} \mathrm{O}$ masers but no continuum emission could be associated with protostellar outflows. The emission we have found could belong to this new class of maser.

\section{Conclusions and future work}

The maser model developed for SNRs indicate that these masers are produced in the presence of a $\mathrm{C}$-type shock. The shocks in $\mathrm{HH}$ objects have a wide range of physical conditions and hence it is possible that some of them could support shock-excited 1720$\mathrm{MHz} \mathrm{OH}$ masers. In SNRs soft x-rays are required to dissociate $\mathrm{H}_{2} \mathrm{O}$ behind the C-shock to produce a sufficient $\mathrm{OH}$ column density to support $1720-\mathrm{MHz}$ masers. The intensity and spectrum of x-rays associated with $\mathrm{HH}$ objects still needs to be investigated. Most observed SNR masers lie along the edge of the shock where the maser path length is maximized. Because the jets in $\mathrm{HH}$ objects do not necessarily lie in the plane of the sky, detection rates could be limited.

In our $\mathrm{OH}$ survey towards $\mathrm{HH}$ objects we have not found any unambiguous maser detections but we have identified several potential $1720-\mathrm{MHz}$ and mainline $\mathrm{OH}$ masers. Follow-up observations of these sources are required to confirm the presence of masers. If any $\mathrm{OH}$ masers are found to be associated with outflows, they should provide useful constraints on the physical conditions in the regions where they occur, and will be used in hydrodynamic models to try and understand what physical and chemical processes are shaping these regions.

\section{References}

Argon, A. L., Reid, M. J. \& Menten, K. M. 2003, ApJ 593, 925

Foster, J. R. \& Caswell, J. L. 2000, ApJ 530, 371

Lockett, P., Gauthier, E. \& Elitzur, M. 1999, ApJ, 511, 235

Reipurth, B. 1999 A General Catalogue of Herbig Haro Objects 2nd Edition, http://casa.colorado.edu/hhcat

Smith, M. D., Khanzadyan, T. \& Davis, C. J. 2003, ApJ 339, 524

Wouterloot, J. G. A., Brand, J. \& Fiegel, K. 1993, A\&AS 98, 589 